\title{
Efeito da posição da omoplata na força máxima isométrica de flexão do ombro
}

\author{
Otília Sousa $^{1}$ \\ Fernando Ribeiro ${ }^{2}$ \\ Mário Leite ${ }^{1}$ \\ Francisco Silva ${ }^{1}$ \\ Ana Paula Azevedo ${ }^{1}$
}

https://doi.org/10.5628/rpcd.07.02.183

\author{
${ }^{1}$ Departamento de Ortofisiatria \\ Serviço de Fisiatria \\ Hospital Geral de Santo António \\ Porto \\ Portugal \\ ${ }^{2}$ Centro de Investigação em Actividade Física, \\ Saúde e Lazer \\ Faculdade de Desporto \\ Universidade do Porto \\ Portugal
}

\begin{abstract}
Effect of scapular position on shoulder flexion maximal isometric strength
\end{abstract} Pelo crescente ênfase colocado na avaliação, prevenção e reabi-
litação de disfunções da omoplata e pela importância atribuída à omoplata e aos seus músculos motores na biomecânica do ombro, os objectivos do nosso estudo foram avaliar o efeito das posições de abdução e adução da omoplata na força máxima isométrica de flexão do ombro medida no plano sagital, e testar a hipótese de que esta é significativamente menor nas posições de adução e abdução comparativamente à posição neutra.

Participaram no estudo dezoito sujeitos aparentemente saudáveis (10 mulheres e 8 homens), com idade compreendida entre os 20 e 24 anos. Os sujeitos realizaram 3 contracções isométricas máximas de flexão do ombro a $90^{\circ}$ no plano sagital com a omoplata em posição neutra, abdução e adução. Para comparação dos valores médios de força isométrica foi utilizada a análise de variância a um factor. A força isométrica foi significativamente superior na posição neutra da omoplata quando comparada com a força medida com a omoplata na posição de abdução $(10,09 \pm 2,41$ vs.8,14 $\pm 2,31 ; p=0,047)$ e adução

$(10,09 \pm 2,41$ vs. $8,11 \pm 2,31 ; p=0,043)$. Os nossos resultados demonstraram que a produção de força isométrica de flexão do ombro é significativamente reduzida nas posições de abdução e adução da omoplata comparativamente à posição neutra.

Palavras-chave: ombro, posição da omoplata, força máxima isométrica
Because of the increased emphasis on assessing, preventing and rehabilitating scapular dysfunctions and because of the great importance given to scapula and to scapular muscles in the shoulder kinetics, the purposes of our study were to assess the effect of scapular abduction and scapular adduction on maximal isometric shoulder elevation strength measured in the sagittal plane, and to test the hypothesis that isometric shoulder strength would be significantly less in the scapular abdution and adduction positions relative to the neutral position. Eighteen healthy volunteers ( 8 men, 10 women) ages 20 to 24 years were recruited to participate in the study. Subjects completed 3 maximal isometric shoulder elevation contractions at $90^{\circ}$ of sagittal plane elevation in the scapular neutral, abduction and adduction positions. Mean isometric strength values were compared using One-way anova. Isometric strength was significantly higher for the neutral position compared with the scapular abduction $(10,09 \pm 2,41$ vs. $8,14 \pm 2,31 ; p=$ $0,047)$ and adduction positions $(10,09 \pm 2,41$ vs. $8,11 \pm 2,31 ; p=$ $0,043)$. Our results showed that the production of shoulder flexion isometric strength is significantly lower in scapular abduction and adduction positions compared with scapular neutral position.

Key-Words: shoulder, scapular position, maximal isometric strength 


\section{INTRODUÇÃO}

Dor e disfunção do complexo articular do ombro são causas comuns em sujeitos que recorrem aos serviços de saúde. Nos últimos anos, têm-se dado particular ênfase ao papel da omoplata e dos seus músculos motores na génese e perpetuação da patologia do ombro. A omoplata desempenha um importante papel na funcionalidade do complexo articular do ombro, sendo a ponte de ligação que assegura a transferência de forças de alta intensidade dos membros inferiores e tronco para os membros superiores (7). É também um ponto de ancoragem de vários músculos (1), uns com actuação na omoplata e outros com actuação ao nível da articulação glenoumeral.

Alterações na função dos músculos estabilizadores da omoplata podem-se traduzir em alterações na sua posição de repouso, condicionando desta forma todos os parâmetros de movimento do complexo articular do ombro. Diminuição da força dos músculos motores da omoplata pode exercer efeito deletério na sua cinemática (1), alterando a função do ombro e o seu centro de rotação instantânea e desta forma conduzir à lesão e à incapacidade $(7,11)$. Alguns autores $(2,3,7)$ têm relacionado as disfunções da omoplata, fisiologia e biomecânica anormal induzida por comprometimento da sua função muscular, com a predisposição para lesões de sobrecarga do ombro.

Pelo crescente ênfase colocado na avaliação, prevenção e reabilitação de disfunções da omoplata e pela importância que lhe é atribuída e aos seus músculos motores na biomecânica do ombro, foi realizado um estudo para investigar a relação entre a posição da omoplata e a função muscular do ombro. Desta forma, os objectivos do estudo foram: examinar o efeito da posição de abdução e de adução da omoplata na força máxima isométrica de flexão do ombro medida no plano sagital; e testar a hipótese de que a força é significativamente menor nas posições de adução e abdução comparativamente à posição neutra. Esta hipótese baseiase na suposição de que as posições de adução e abdução da omoplata ao alterarem a relação de tensãoalongamento dos seus estabilizadores mediais (músculo trapézio e rombóides) induzem uma redução na efectividade do par déltoide-coifa dos rotadores no movimento de flexão do ombro.

\section{MATERIAL E MÉTODOS}

\section{Participantes}

A amostra foi constituída por 18 sujeitos aparentemente saudáveis, 10 do sexo feminino e 8 do sexo masculino, com idade compreendida entre os $20 \mathrm{e}$ 24 anos (idade média \pm desvio padrão: $22,2 \pm 1,4$ anos). Os sujeitos foram recrutados por conveniência na Escola Superior de Tecnologias da Saúde do Porto. Para inclusão no estudo os sujeitos deveriam apresentar amplitude articular do ombro completa (flexão $180^{\circ}$; rotação externa $90^{\circ}$; rotação interna $70^{\circ}$ ), sem dor e simétrica, e não apresentar nenhum dos seguintes critérios de exclusão: dor actual na região cervical ou ombro; história de fractura, luxação ou cirurgia na região cervical, torácica e ombro; escoliose ou cifose dorsal superior a $30^{\circ}$; patologia degenerativa ou congénita da coluna cervical; alteração congénita da omoplata; patologia neuromuscular conhecida; instabilidade do ombro conhecida ou suspeita; com o membro superior na posição anatómica a omoplata apresentar-se em abdução ou adução; história de patologia cardiopulmonar; gravidez (11). Todos os sujeitos completaram a recolha de dados numa única sessão matinal, após familiarização com o protocolo experimental e com os instrumentos de avaliação. Foi testado o membro superior dominante, o direito em todos os sujeitos. Todos os sujeitos deram o seu consentimento informado por escrito e todos os procedimentos foram efectuados de acordo com a declaração de Helsínquia. O estudo foi aprovado pela comissão de ética local.

\section{Avaliação da força muscular dos flexores do ombro}

A força máxima isométrica dos flexores do ombro foi medida com recurso a um dinamómetro (Globus Ergo Meter, Globus Itália) em três posições diferentes da omoplata: posição neutra, abdução e adução. Foi pedido aos sujeitos para não praticarem actividade física com o tronco superior nas 48 horas precedentes à avaliação. Todos os testes de avaliação da força muscular foram realizados pelo mesmo investigador. $\mathrm{Na}$ posição de teste o sujeito estava sentado numa cadeira, sob uma gaiola de Rocher, com encosto rígido e sem limitação dos movimentos de abdução e adução da omoplata. O tronco foi imobilizado ao encosto da cadeira por meio de bandas estabilizadoras para evitar movimentos de flexão/extensão e/ou rotação do tronco durante a avaliação. Todos os testes de ava- 
liação da força muscular foram efectuados com o membro superior posicionado a $90^{\circ}$ de elevação no plano sagital, ou seja $90^{\circ}$ de flexão, com o cotovelo em extensão e a mão fechada. Apesar do plano da omoplata permitir uma medição mais funcional, o plano sagital foi escolhido para permitir maior simplicidade na determinação das três posições da omoplata, pela maior amplitude de movimento de abdução da omoplata disponível e pela definição de abdução da omoplata mais comummente usada determinar a posição com o membro superior a 90 de elevação no plano sagital. O membro foi suportado por uma suspensão axial, permitindo a manutenção da posição de teste e o descanso do membro durante os períodos de repouso. A célula de força do dinamómetro foi fixada ao sujeito e à gaiola de Rocher por um sistema que permitia redireccionar a linha de tensão quando o sujeito obtinha as diferentes posições da omoplata, mantendo um ângulo de $90^{\circ}$ entre o membro do sujeito e o dinamómetro durante as 3 posições de teste. As três posições de teste da omoplata (neutra, abdução e adução) foram obtidas activamente pelos sujeitos. A posição neutra foi definida como a posição de repouso mais confortável entre os extremos de abdução e adução activas, subjectivamente determinadas pelos sujeitos. As posições de abdução e adução foram determinadas como sendo a máxima adução e abdução que os sujeitos eram capazes de obter e manter activamente, tendo como distância mínima da posição neutra $5 \mathrm{~cm}$ para a abdução e 3 $\mathrm{cm}$ para a adução (Figura 1), sendo a extremidade distal do terceiro metacarpo o ponto de referência. A posição do membro superior e tronco foi confirmada com recurso a um goniómetro universal, tendo as distâncias de abdução e adução da omoplata sido confirmadas através de uma fita métrica.
Antes da avaliação da força máxima isométrica os sujeitos realizaram um breve aquecimento composto por 5 movimentos de amplitude total do ombro em todas as direcções e por 2 contracções isométricas sub-máximas durante cinco segundos na posição de $90^{\circ}$ de elevação do ombro no plano sagital para cada uma das três posições da omoplata avaliadas. Após um minuto de repouso, foi pedido aos sujeitos que realizassem 3 contracções isométricas máximas, com 5 segundos de duração, para cada uma das posições de teste da omoplata, sendo escolhido para análise o valor mais elevado resultante das três medições. O tempo de repouso entre contracções foi de um minuto e o tempo de repouso entre posições de teste foi de dois minutos, repousando o membro superior suspenso pela suspensão axial.

Para minimizar os efeitos da fadiga e de aprendizagem sobre a última posição de teste, cada uma das possíveis sequências das posições de teste foi efectuada a igual número de sujeitos. Durante o teste a posição do sujeito foi constantemente monitorizada por um fisioterapeuta. Após a mudança de posição da omoplata todos os ângulos eram verificados e o dinamómetro isométrico ajustado, para assegura consistência na posição de teste.

\section{Análise Estatística}

Foi utilizada a estatística descritiva para calcular a média e o desvio padrão dos valores da força isométrica em cada uma das 3 posições de teste da omoplata. Foi utilizado o teste de Shapiro-Wilk para testar a normalidade de distribuição dos dados. Para comparação de médias foi utilizada a análise de variância a um factor. O teste de Bonferroni foi usado para identificar entre que posições de teste se verificaram diferenças significativas. O nível de significância foi estabelecido em $5 \%$.
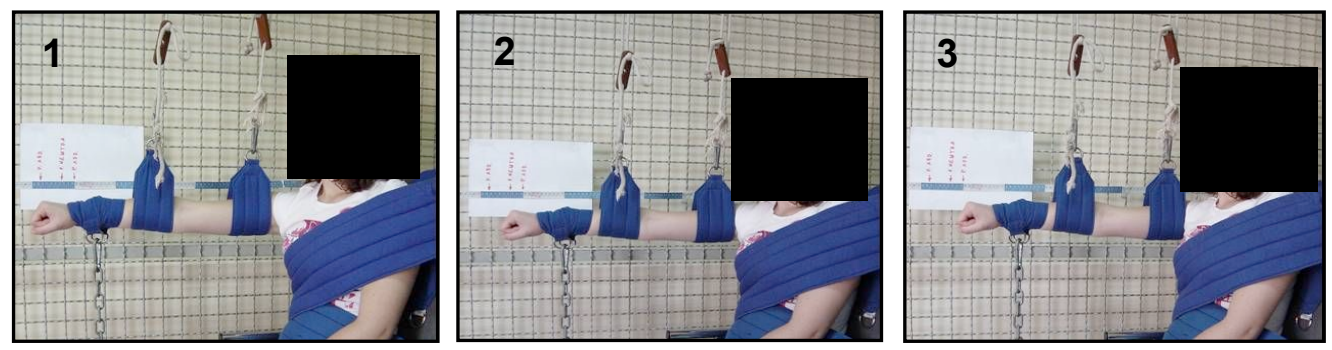

Figura 1. Sujeito na posição de teste com a omoplata em posição de abdução (1), posição neutra [2] e adução [3] 


\section{RESULTADOS}

Todos os sujeitos que participaram no estudo completaram com sucesso as tarefas que lhes foram determinadas.

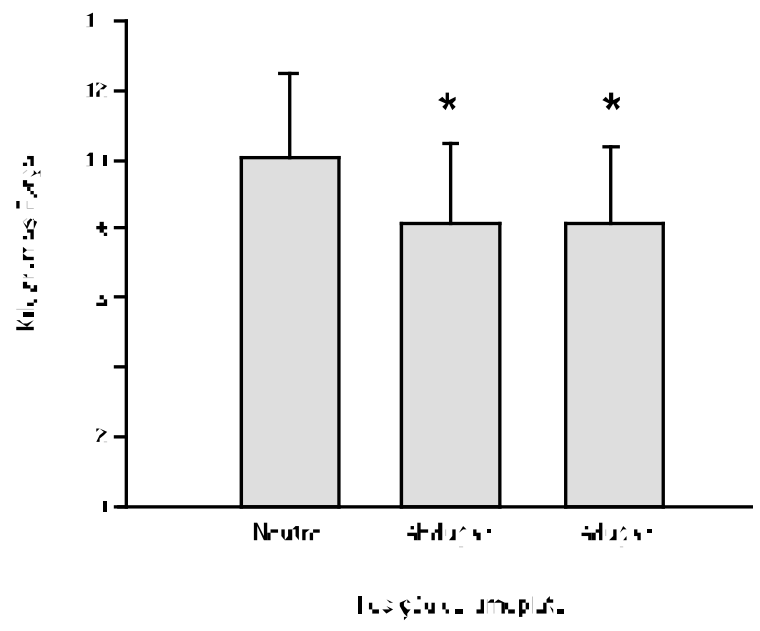

* diferença estatisticamente significativa para a posição neutra; $p \leq 0,05$ Figura 1. Média e desvio padrão da força máxima isométrica para as posições da omoplata testadas

A força máxima isométrica dos flexores do ombro é significativamente superior na posição neutra da omoplata quando comparada com a força medida com a omoplata na posição de abdução $(10,09 \pm$ $2,41$ vs. $8,14 \pm 2,31 ; p=0,047)$ e adução $(10,09 \pm$ $2,41$ vs. $8,11 \pm 2,31 ; \mathrm{p}=0,043)$. Não se verificaram diferenças estatisticamente significativas entre a força máxima isométrica produzida pelos flexores do ombro com a omoplata na posição de adução e na posição de abdução (Figura 1 e Quadro 1).

A produção de força isométrica pelos flexores do ombro com a omoplata em abdução e adução é aproximadamente $20 \%$ inferior à força produzida quando a omoplata se encontra na posição neutra (Quadro 1).

Quadro 1. Valores de força máxima isométrica $[\mathrm{Kg}]$ e percentagem de diminuição comparativamente à posição neutra

\begin{tabular}{lccc}
\hline $\begin{array}{l}\text { Posição da } \\
\text { omoplata }\end{array}$ & $\mathrm{n}$ & $\begin{array}{c}\text { Força máxima } \\
\text { isométrica }\end{array}$ & $\begin{array}{c}\text { \% de } \\
\text { diminuição }\end{array}$ \\
\hline Neutra & 18 & $10,09 \pm 2,41$ & - \\
Abdução & 18 & $8,14 \pm 2,31^{*}$ & $19,82 \pm 9,55$ \\
Adução & 18 & $8,11 \pm 2,31^{* *}$ & $19,97 \pm 9,05$ \\
\hline
\end{tabular}

* diferença estatisticamente significativa para a posição neutra; $p=0,047$ ** diferença estatisticamente significativa para a posição neutra; $p=0,043$

\section{DISCUSSÃO}

Este estudo vem na linha dos estudos iniciados em 2002 por Smith e colaboradores (11) na Clínica Mayo, Rochester, Minessota, no qual se verificou que a função muscular do ombro é significativamente alterada pela posição da omoplata. Os resultados do corrente estudo indicam que a posição da omoplata altera significativamente a função muscular do ombro na posição de $90^{\circ}$ de flexão, suportando a nossa hipótese de que a força produzida pelos flexores do ombro é significativamente menor nas posições de adução e abdução da omoplata comparativamente à posição neutra. Tendo-se verificado uma diminuição de cerca de $20 \%$ na produção de força isométrica pelos flexores do ombro com a omoplata em posição de adução e abdução comparativamente à posição neutra.

Utilizando um protocolo idêntico ao usado neste estudo, Smith et al. (11) reportaram redução de $30 \%$ na força máxima isométrica dos flexores do ombro com a omoplata em adução e $23 \%$ com a omoplata em abdução. Da mesma forma Kebaetse et al. (5) encontraram uma redução de $16 \%$ da força máxima isométrica de abdução em sujeitos saudáveis com uma postura ligeiramente cifótica; a medição da força foi efectuada a $90^{\circ}$ de elevação do ombro no plano escapular. Recentemente, Smith et al. (10) verificaram que a posição de abdução da omoplata reduzia significativamente a força isométrica dos rotadores internos e externos do ombro em diversos ângulos articulares. Estes dados indicam que a função muscular do ombro pode estar comprometida quando a omoplata se desvia da sua posição neutra. Os resultados deste estudo reforçam a importância da posição da omoplata na função e na reabilitação do complexo articular ombro tal como sugerido em estudos anteriores $(6,7,9,13)$.

O desvio da omoplata, em relação à sua posição neutra, pode comprometer a capacidade desta fornecer uma base de suporte estável e colocar os músculos estabilizadores da mesma em posição desvantajosa na sua curva de comprimento- tensão (10-12). De facto, a modificação na relação comprimento-tensão ou sinergias musculares verificadas no desvio da omoplata da posição neutra, parece ser uma hipótese plausível para explicar os resultados deste estudo. Ellenbecker e Mattalino (4) demonstraram que a força muscular do ombro é alterada em função do 
plano em que este está posicionado. Da mesma forma que a posição de abdução e adução da omoplata induz desvantagem nos seus músculos estabilizadores, o desvio desta da sua posição neutra também reduz a capacidade de gerar força por parte da coifa dos rotadores, comprometendo desta forma a geração de força de todo o complexo articular do ombro $(7,8)$. Neste caso particular o desvio da omoplata da posição neutra parece reduzir a eficácia do par deltóide-coifa dos rotadores durante o movimento de flexão do ombro (11).

Este estudo apresenta algumas limitações: (i) foram investigados jovens aparentemente saudáveis sem alterações no complexo articular do ombro. A opção foi feita para diminuir a presença de factores clínicos potencialmente confundidores tais como a etiologia do sintoma, a duração e a severidade; (ii) a avaliação isométrica da força muscular pode não se correlacionar com a maioria das actividades da vida diária e com as actividades laborais. No entanto, face ás opções disponíveis, este método de avaliação da força muscular foi o que se revelou mais útil na realização deste estudo; (iii) a posição da omoplata relativamente ao seu movimento de rotação superior/inferior não foi objectivamente monitorizada. Apesar da posição inicial do membro superior e do tronco ter sido confirmada com recurso a um goniómetro universal, sugere-se que futuros estudos monitorizem objectivamente os movimentos de rotação superior/inferior da omoplata.

Apesar das limitações do nosso estudo os nossos resultados indicam que a força isométrica máxima dos flexores do ombro, a $90^{\circ}$ de flexão, é dependente da posição da omoplata. É importante realçar que o efeito deletério exercido pela posição da omoplata na capacidade de gerar força foi similar para a posição de abdução e adução. Estes resultados têm importantes implicações para todos aqueles que previnem, avaliam e tratam patologias do ombro. Especial atenção deve ser dada ao impacto que a posição da omoplata exerce na função dos músculos do ombro.

Várias actividades desportivas, tais como a natação, voleibol, ténis, andebol, colocam o complexo articular do ombro em posições vulneráveis possibilitando a ocorrência de patologias inerentes à realização de esforços repetidos. A diminuição na produção de força de flexão do ombro induzida por alterações na posição de repouso da omoplata poderá desta forma, para além de predispor a lesões de sobre-uso $(6,7)$, diminuir a qualidade e eficiência do gesto desportivo. Os resultados deste estudo realçam a importância dos profissionais da área das ciências do desporto e educação física na prevenção de alterações na posição de repouso da omoplata secundárias à diminuição da capacidade muscular dos seus músculos estabilizadores, procurando não só optimizar o gesto técnico como também prevenir a ocorrência de lesões. Da mesma forma, em contexto clínico, a avaliação funcional do complexo articular do ombro realizada por médicos e fisioterapeutas a atletas de modalidades que incluem frequentemente movimentos das extremidades superiores acima da cabeça, deverá contemplar a avaliação da posição de repouso da omoplata.

Futuros estudos são necessários para elucidar sobre o impacto da posição da omoplata na função muscular do ombro em sujeitos com patologia sintomática do ombro, bem como em atletas de modalidades nos quais o uso do membro superior seja preponderante. Este estudo demonstrou que a produção de força isométrica de flexão do ombro é significativamente reduzida nas posições de abdução e adução da omoplata comparativamente à posição neutra.

\section{AGRADECIMENTOS}

Este estudo foi parcialmente apresentado nas $1^{\mathrm{a}} \mathrm{s}$ Jornadas de Fisioterapia em Saúde Ocupacional, que se realizaram de 17 a 18 de Fevereiro de 2006, em Coimbra, Portugal, sendo de inteira justiça agradecer as contribuições que nessa ocasião foram feitas para a melhoria da redacção final deste trabalho.

\section{CORRESPONDÊNCIA}

Fisioterapeuta Otília Sousa

Serviço de Fisiatria, Hospital Geral de Santo António

Largo Prof. Abel Salazar

4099-001 Porto, Portugal

e-mail: fisiootilia@gmail.com 


\section{REFERÊNCIAS}

1. Cools AM, Witvrouw EE, Declercq GA, Vanderstraeten GG, Cambier DC (2004). Evaluation of isokinetic force production and associated muscle activity in the scapular rotators during a protraction-retraction movement in overhead athletes with impingement symptoms. Br J Sports Med 38(1): 64-68

2. Doukas WC, Speer KP (2001). Anatomy, pathophysiology, and biomechanics of shoulder instability. Orthop Clin N Am 32(3): 381-391

3. Ellen MI, Gilhool JJ, Rogers DP (2000). Scapular instability. The scapulothoracic joint. Phys Med Rehabil Clin N Am 11(4): 755-770

4. Ellenbecker TS, Mattalino AJ (1997). Concentric isokinetic shoulder internal and external rotation strength in professional baseball pitchers. J Orthop Sports Phys Ther 25(5): 323-328

5. Kebaetse M, McClure P, Pratt NA (1999). Thoracic position effect on shoulder range of motion, strength, and three-dimensional scapular kinematics. Arch Phys Med Rehabil 80(8): 945-950

6. Kibler WB (1991). The role of the scapular in overhead throwing motion. Contemp Orthop 22(5): 525-532

7. Kibler WB (1998). The role of scapula in athletic shoulder function. Am J Sports Med 26(2): 325-337

8. Kibler WB, McMullen J, Uhl T (2001). Shoulder rehabilitation strategies, guidelines and practise. Orthop Clin North Am 32(3): 527-538

9. Paine RM, Voight M (1993). The role of the scapula. J Orthop Sports Phys Ther 18(1): 386-391

10. Smith J, Dietrich CT, Kotajarvi BR, Kaufman KR (2006). The effect of scapular protraction on isometric shoulder rotation strength in normal subjects. J Shoulder Elbow Surg 15(3): 339-343

11. Smith J, Kotajarvi BR, Padgett DJ, Eischen JJ (2002). Effect of scapular protraction and retraction on isometric shoulder elevation strength. Arch Phys Med Rehabil 83(3): 367370

12. Su C-Y, Lin J-H, Chien T-H, Cheng K-F, Sung Y-T (1994). Grip strength in different positions of the elbow and shoulder. Arch Phys Med Rehabil 75(7): 812-815

13. Wilk KE, Arrigo C (1993). Current concepts in the rehabilitation of the athletic shoulder. J Orthop Sports Phys Ther 18(1): 365-378 